\title{
EL PROFESORADO DE DIBUJO EN LA ESCUELA NORMAL № 2 (ROSARIO, 1935 - 1949): UNA ESCUELA DE "FORMACIÓN ESTÉTICA PARA EL FUTURO NIÑO ARGENTINO"
}

\author{
María Elisa Welti (Universidad Nacional de Rosario)* \\ elisawelti@hotmail.com
}

Recibido: 22/07/2011 Aceptado: 14/11/2011

\section{Resumen}

En este trabajo nos proponemos indagar la articulación entre nuevas vertientes estéticas y corrientes pedagógicas renovadoras que tiene lugar en las primeras décadas del siglo XX en la Escuela Normal $\mathrm{N}^{\circ} 2$ (actual Escuela Normal Superior $\mathrm{N}^{\circ} 35$ ) de la ciudad de Rosario y que favorece la creación del Profesorado de Dibujo en el nivel superior de esa institución.

Situamos esta iniciativa de formación de profesores especializados en la enseñanza del dibujo en el contexto local y los debates de la época. Asimismo, analizamos la intersección que en ella se evidencia entre las expresiones locales del movimiento escolanovista y la intensa agitación que genera por entonces la irrupción de las vanguardias artísticas e intelectuales en la ciudad. En tal sentido, resulta clave considerar el protagonismo que tuvieron en la apertura de esta carrera dos figuras destacadas de la época, como Angel Guido y Dolores Dabat.

Creemos que el análisis de esta primera experiencia de formación de profesores de dibujo inscripta en una escuela normal ofrece numerosos elementos que aportan a la construcción de la historia, aún escasa, de la educación artística en la ciudad de Rosario y, específicamente, de la formación de docentes en esa área.

\section{Palabras clave}

Dibujo - Profesorado - Educación Artística - Rosario - Escuela Normal.

* Magister en Educación con mención en Formación Docente. Universidad Nacional de Entre Ríos. Profesora en Ciencias de la Educación. Universidad Nacional de Rosario. 


\section{Abstract}

In this paper, we intend to explore the link between the recent aesthetic strands and innovative pedagogical trends that takes place in the first decades of the twentieth century in Normal School $N^{\circ} 2$ (today's Normal School $N^{\circ} 35$ ) in the city of Rosario and that leads to the creation of the Drawing Teacher Training Course as part of the post-secondary education provided by the institution.

In this paper, we place this initiative to train teachers specialized in the teaching of drawing in the local context and in the debates of the time. We also explore the evident intersection between the local strands of the progressive education movement and the intense agitation generated by the emergence of the artistic and intellectual avant-garde movements in the city. In this regard, it is crucial to consider the major role played by two leading figures of the time, Angel Guido and Dolores Dabat, in the creation of this post-secondary course.

We believe that the analysis of this first experience of training drawing teachers in a normal school offers numerous elements that contribute to the construction of the still short history of artistic education in the city of Rosario and specifically of teacher training in that area.

\section{Key words}

Education.

Drawing - Teacher training College - Normal School - Rosario - Artistic

\section{Introducción}

Las primeras décadas del siglo $X X$ revisten un carácter fundacional en el campo de la cultura y la educación, son años en los que se percibe el tránsito entre lo que era y lo que vendrá, en los que todo "está como por ser" (Funes, 2006). En esas décadas, educadores, intelectuales y artistas de la ciudad de Rosario generaron un intenso movimiento que dio lugar a la creación y consolidación de buena parte de las instituciones y agencias vinculadas al arte, la educación y la cultura locales. Entre ellas se encuentra la apertura a mediados de la década del 30, en la Escuela Normal $N^{\circ} 2$, del Profesorado de Dibujo al que nos referimos en este escrito.

El análisis de la gestación y devenir de esta carrera constituye una clave de importancia para la comprensión del proceso de institucionalización de la educación artística en el ámbito local (1); proceso que se lleva a cabo de modo simultáneo y articulado, tanto con la expansión del sistema educativo oficial y de la preparación profesional de los docentes, como con la progresiva autonomización del campo de la producción artística, correlativa con la creación y afianzamiento de espacios públicos de circulación del arte y la consiguiente especialización de la formación de artistas. Además, en la institución que da origen de esta experiencia pionera de formación de docentes de dibujo, conflu- 
yen la matriz normalista y el influjo escolanovista de corte renovador que signó el campo pedagógico de la época.

El Profesorado de Dibujo constituyó el primero en su tipo en la ciudad y, dada la casi inexistente oferta de formación artística especializada en el marco del sistema educativo público local hasta principios de la década del 40, ocupó en buena medida ese lugar posicionándose también como espacio de formación de artistas.

Hasta entonces, la formación artística en la ciudad de Rosario sólo se desarrollaba en ámbitos privados, mayormente a cargo de artistas europeos radicados en la ciudad: tal es el caso del Instituto de Bellas Artes "Doménico Morelli" fundado en 1906 y dirigido por el pintor y escenógrafo italiano Mateo Casella; poco después se fundó la Academia "Fomento de las Artes" del italiano Ferruccio Pagni; casi simultáneamente comenzó a funcionar la academia del alemán Enrique Schwender; en 1907 el catalán Enrique Munné abrió su academia; otro pintor español, Eugenio Fornells, comenzó a enseñar en 1908; el mismo año Fernando Gaspary, de origen francés, abrió su academia en la ciudad. Algunos de estos artistas, como Munné, Schwender y Gaspary fueron también profesores de dibujo de las escuelas secundarias locales (Slullitel, 1968; Sendra, 1990; Córdoba Lutges, 1972).

Por otra parte, la consolidación del campo artístico local se opera en esos años a través de la formalización en la ciudad de los primeros espacios destinados a la difusión artística, entre los que se destacan: la sede local de la galería de arte Witcomb (1918); la Biblioteca Argentina (1913) que, bajo la dirección de Juan Álvarez, constituyó un ámbito de exposiciones, conciertos y conferencias; los Salones de Otoño (1917); el Museo Municipal de Bellas Artes (1920).

En este contexto de creciente autonomización de los espacios de circulación del arte local, la carencia de instituciones oficiales que atendieran la formación especializada de los artistas constituía un reclamo por parte de diversos sectores. Fueron algunas de las primeras agrupaciones de artistas -entre las que se cuentan Nexus y la Mutualidad de Artistas Plásticos- las que comenzaron a solicitar a las autoridades provinciales la instalación de una institución de estas características. A pesar de estas demandas, el establecimiento requerido demoraría aún años en comenzar a funcionar (2).

Es en este escenario expectante en el que desde la Escuela Normal $N^{\circ} 2$, especializada en la formación de maestras, se gestiona la apertura de este Profesorado de Dibujo -junto al Profesorado de Música- que cubre transitoriamente la vacancia existente en la formación local de artistas. Veremos ahora las condiciones institucionales que hicieron posible la apertura de esta carrera, algunas de sus particularidades y su devenir hasta mediados del siglo XX. 


\section{La escuela y su "tarea de cultura": las condiciones y el contexto para la formación artística}

Ahora bien, ¿por qué una escuela normal abocada a la preparación del magisterio primario albergó estas carreras de formación docente especializadas en música y dibujo? La historia de la escuela ofrece algunas pistas para abordar esta cuestión. Recordemos que se crea a principios de 1910. Martín Herrera, egresado de la Escuela Normal de Paraná, estuvo a cargo de su dirección hasta 1920. En 1924, asume la dirección la maestra y pedagoga Dolores Dabat oriunda de la ciudad de Paraná y también graduada de la Escuela Normal de esa ciudad.

En las investigaciones histórico-educativas esta institución es reconocida fundamentalmente por las experiencias escolanovistas que en ella -y a partir de ella- se realizan desde poco después de su creación: la Escuela al Aire Libre, a cargo de las primeras egresadas del magisterio en un barrio periférico de la ciudad, y los ensayos decrolyanos que tienen lugar en la misma escuela normal durante las décadas del 20 y del 30 (3). De la misma manera, son reconocidas como figuras claves dentro del campo de la pedagogía vernácula los directores ya mencionados, Martín Herrera y Dolores Dabat.

Cabe apuntar que el escolanovismo ingresa al país en esos años y se instala, luego de una primera etapa de apropiación lenta y gradual, en el escenario pedagógico argentino que, a su vez, lo resignifica y lo articula con el proceso complejo de modernización cultural que se estaba operando por entonces, dando lugar a particulares lazos entre el movimiento de renovación pedagógica y las tendencias estéticas que le eran contemporáneas. De hecho, en buena parte de las experiencias escolanovistas locales se evidencia un sesgo fuertemente estético que se presenta como "elemento de identificación individual, colectivo y social" (Carli, 1992, p. 137) (4).

En el caso de la escuela normal que nos ocupa, esta implicación entre renovación pedagógica e inquietudes estéticas asume cualidades particulares puesto que se trata de una institución que se inscribe además con notable fuerza en la trama intelectual y cultural de la época. La vinculación de la escuela con la vida cultural local es fundamental para comprender cómo se gesta la apertura de las carreras de formación de profesores en artes.

Un ejemplo clave para entender la relevancia de la institución en la ciudad lo constituye la fundación de la Universidad Popular de Rosario en 1918, cuya primera y principal sede física se localiza en el edificio en la escuela normal (5). Esta proximidad edilicia es fruto de una colaboración que se extiende en el tiempo y que da lugar a la realización de actividades conjuntas. En la Universidad Popular se dictaron, entre otros, numerosos cursos de formación artística, entre los que se destacan el curso de tallado y cerámica a cargo del señor Rogelio Segovia, el curso de fotografía práctica y artística dictado por el señor Orfeo Berizzo, el curso de dibujo lineal a cargo de Deolindo Saccone, el curso 
de dibujo ornamental a cargo del pintor Enrique Munné y el curso de estética musical dictado por Eva Moller. Algunos de estos cursos fueron dictados por profesores de la Escuela Normal -y de hecho era en ella en donde habían tenido su origen- como el curso de instrucción literaria a cargo de Bernardina Dabat primero y de Ernesta Robertaccio más tarde, el curso de canto y música coral desarrollado por J. Berrini o el curso de dibujo y artes decorativas dictado por Lelia Echezarreta (Memoria del Instituto Social 1928 - 1932, 1934).

Por otra parte, la escuela fue el núcleo desde el cual se conformaron asociaciones de egresadas y de padres que intervinieron activamente en la escena cultural y pedagógica local. Estas asociaciones realizaron numerosas actividades que generaron el reconocimiento del medio, prueba de ello es una nota publicada en 1932 en el periódico "La Tierra":

Esa Escuela Normal $N^{\circ} 2$, por el órgano externo de gran alcance que ella misma se ha creado, las asociaciones de padres y ex-alumnas, ha puesto en contacto a la ciudad con los más destacados intelectuales del país y no pocos del extranjero; ha organizado conciertos y otras actuaciones artísticas de valores de primera línea en Rosario y fuera de Rosario, y en esa persistente tarea de cultura y en esa perenne tensión de simpatía humana, intensifica diariamente su labor y amplía su radio de influencia (La Tierra, 7 de julio de 1932).

Así se destaca la profusa cantidad de conferencias y eventos desarrollados en la escuela y organizados por las asociaciones referidas de los que participaron artistas, intelectuales y pedagogos reconocidos tales como Anibal Ponce, el crítico de arte Jorge Romero Brest, el especialista en historia del arte José León Pagano, las poetisas Alfonsina Storni y Gabriela Mistral, los escritores Eduardo Mallea, Ramón Gómez de la Serna, Rafael Alberti y Enrique Gonzalez Tuñón, la actriz Lola Membrives, los pedagogos Adolfo Ferriere, Ernesto Nelson, Lorenzo Luzuriaga, Juan Mantovani y Olga Cossettini.

Otra clara expresión de este protagonismo la constituye la publicación periódica de esas asociaciones, la revista Quid Novi?, dirigida por Dolores Dabat, en la se registra la participación de intelectuales y artistas notables y que se inscribe estéticamente en el clima de la época (6).

La asociación de ex-alumnas gestionó además un proyecto concebido por una de sus integrantes y luego presidenta de la agrupación, la escritora Ana María Benito: la realización de un Cinematógrafo de Arte, inaugurado en junio de 1930, en el que se realizaban funciones periódicas con un programa de cine de vanguardia proporcionadas por el Cine Club de Buenos Aires. Así lo expresa la misma Dabat remitiéndose a las Memorias de la asociación de ex-alumnas: "Se trataba de producciones de vanguardia de procedencia europea y norteamericana, excluidas de los programas habituales de los cinematógrafos por el hecho de constituir espectáculos para públicos selectos" (Dabat, 1935, p. 359). Entre las películas proyectadas se contaron, por ejemplo, las del reconocido director de cine ruso Sergei Eisenstein "Octubre" (1927) y "La línea general" (1929) o "El gabinete del Doctor Caligari", película muda dirigida en 1920 por 
Robert Wiene, considerada como pionera del cine expresionista alemán. La máquina de proyección fue facilitada para esta actividad por la Asociación de Padres que la había adquirido en 1929 a pedido de la dirección de la escuela (Dabat, 1935).

Este no es un dato menor si consideramos que el Cine Club de Buenos Aires comienza su funcionamiento poco antes, en 1929, en la sociedad Amigos del Arte -entidad que por entonces nucleaba a buena parte de los intelectuales y artistas porteños de avanzada- a instancias de un grupo de jóvenes entre los que se contaban Jorge Romero Brest, Horacio Cóppola y Jorge Luis Borges (Peña, 2008). La iniciativa constituye un claro indicio de cuáles eran los intereses y las relaciones de al menos algunos de los actores que participaban entonces de la escuela normal y del modo en que esto resulta en beneficio del protagonismo cultural que asume la institución en el ámbito local.

Como vemos, el interés por las actividades vinculadas al arte estaba ya presente desde tiempo antes de la creación formal de los Profesorados de Música y Dibujo, del mismo modo que era clara también la estrecha vinculación entre la escuela y el medio artístico e intelectual. De hecho, el mismo día que se inauguran oficialmente los profesorados lo hacen también la biblioteca y el museo de la institución. Ahora bien, esa biblioteca albergaba algo más que libros, era escenario de la monumental pintura mural que Alfredo Guido, su autor, eligiera donar a la institución (7). Después de numerosas gestiones y de varias reformas, este mural fue instalado en lo que entonces funcionaba como sala de la biblioteca. El mural tiene 200 metros cuadrados pintados al óleo y ofrece escenas coloridas -fauvistas casi- que representan las diversas regiones del país.

En suma, como puede notarse, no son pocas las iniciativas que dotan a la institución de una vitalidad que ampliaba el marco escolar para las innovaciones educativas y que le permitieron asumir proyectos de corte cultural y artístico; vitalidad que es precisamente, desde nuestra perspectiva, la que genera las condiciones y el ambiente que hacen posible que a mediados de la década del 30 se inicie allí una experiencia inédita de formación de docentes en artes.

\section{La formación artística en la escuela normal}

Ya hemos afirmado que buena parte de la vida escolar se vinculaba de uno u otro modo al campo artístico y no poco tenía que ver con ello la sensibilidad de Dolores Dabat. Una ex-alumna relata su paso por la escuela en época de Dabat:

La escuela era como todo lo que rodeaba a ella, hermosa. Muchas alumnas proveníamos de ambientes modestos, de casas humildes; entrábamos a un edificio en que se cuidaba el menor detalle, nada roto ni despintado. Aprendíamos a asignarle importancia al ámbito, al orden, a la estética. Lo infundía con tal profundidad que en mi 
paso por muchas escuelas de campo, de barrios pobres y de otros, lo consideré fundamental; cada cambio que yo lograba me recordaba el empeño de Lola cuando nos señalaba la planta que había colgado, el brote recién nacido (...) Íbamos de excursión en ómnibus, comentando los colores del follaje, y ahí nomás, sin temario ni título nos introdujo al impresionismo y al puntillismo. Nos habló de Fernando Fader, despertó el interés por ver láminas, visitar museos. (Testimonio de una ex-alumna recogido por R. Ziperovich, en Puiggrós, 1992, p. 177)

Es, pues, en este clima estetizante en el que tempranamente comienzan a desarrollarse dentro de la escuela diversas alternativas de formación en esta área. En la década del 20 llega a la escuela con un curso de dibujo Lelia Echezarreta, quien poco después sería autora de numerosas ilustraciones de la revista Quid Novi? y docente del Instituto Social de la UNL y del Profesorado de Dibujo. Echezarreta se especializaba en "dibujo botánico" (8); dibujo acerca del que señala lúcidamente Dabat fue "algo más, fue un documento" y del que agrega:

(...) las imágenes confiadas al recuerdo y la estructura de los vegetales a ojo desnudo o a través de lupas o del microscopio se fueron aclarando y "rectificando" y se trasladaron al papel en rasgos o en colores como expresión de una verdad científica a la que -por añadidura- acompañaba una belleza de ejecución inesperada.

(Dabat, 1935, p. 321)

Se inician por entonces cursos de extensión cultural organizados por la asociación de ex-alumnas entre los que se encontraba un curso de dibujo decorativo a cargo de la mencionada Lelia Echezarreta y otros de formación literaria a cargo de Bernardina Dabat y Ana María Benito. Estos cursos, tal como se anticipara más arriba, fueron pasados en 1930 al ámbito de la Universidad Popular, ya integrada al Instituto Social de la Universidad del Litoral, a pedido de Juan Alvarez quien era por entonces director del mismo, y allí siguieron desarrollándose durante toda la década.

Por otro lado, en 1930 se establece por iniciativa del Dr. Ernesto Padilla, quien asume el Ministerio de Justicia e Instrucción Pública en el marco de la presidencia a Uriburu, un nuevo tipo de escuela intermedia conformada por "cursos vocacionales" preparatorios para oficios que poco después se suprimieron pero que, en este caso, interesa relevar puesto que ratifican la clara inclinación del establecimiento por la formación artística. Se abrieron entonces en la escuela tres cursos vocacionales: de comercio, de artes aplicadas a la industria y de economía doméstica. En esta instancia aparece ya la figura de Angel Guido, más tarde regente del Profesorado de Dibujo, como profesor de dibujo artístico del curso de artes aplicadas a la industria durante 1931 y 1932. 


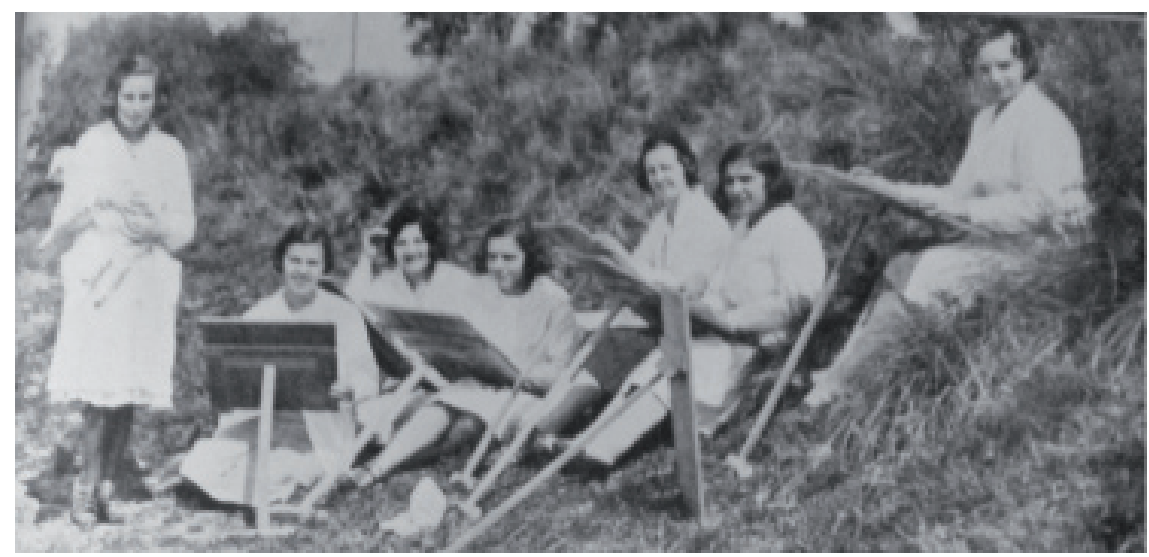

Clase de dibujo a orillas del Saladillo. Fotografía publicada en la revista Quid Novi?, Año 2, N 5, 1933.

\section{El Profesorado de Dibujo: la "formación estética del futuro niño argentino"}

El Profesorado de Dibujo se crea, junto al Profesorado de Música, por decreto del Poder Ejecutivo, el 17 de diciembre de 1935. Ambos se inauguran oficialmente en septiembre de 1936. Si bien se trató de carreras destinadas, al menos en un principio, a "mejorar la cultura" de los maestros y eran ellos los principales destinatarios, era posible el ingreso a las mismas sin contar previamente con el título de magisterio; esto es, a quienes quisieran ingresar y tuvieran un título de bachiller se les requería la aprobación de un examen de Pedagogía. También se exigía a todos los ingresantes demostrar capacidad en la especialidad elegida, para lo cual se debía aprobar un examen de ingreso obligatorio.

Cabe apuntar a modo de antecedente que ya tiempo antes de esta fecha, en 1927, Dolores Dabat había elaborado un proyecto para la apertura en la Escuela de un Profesorado de Estética en el que señalaba:

(...) el arte ha dejado de ser esotérico y suntuario (...) uno de los grandes triunfos de la democracia consiste en haberlo sacado de las manos frías de las academias y de los museos para entregarlo a las escuelas, a los salones particulares, a las exposiciones de libre acceso, a las oficinas del pueblo, en donde habría de encontrar las fuentes no cegadas del sentimiento y la originalidad (Dabat, 1936, p. 9).

De este modo, para Dabat constituye un triunfo en el camino de la democratización del acceso al arte y a la cultura la implantación de un trayecto preparatorio para profesores de artes en una escuela normal, institución que 
considera más próxima a los sectores populares que una "fría" academia o un museo.

La misma Dabat señala que este proyecto tenía como finalidad, en coincidencia con el profesorado que se concreta en 1935, "formar a los profesores que han de enseñar mañana en los cursos normales y secundarios en dos asignaturas tan útiles como interesantes, cuales son el dibujo y la música, dándoles la preparación técnica y docente necesaria para llenar con éxito su misión" (Dabat, 1936, p. 9).

El Profesorado de Dibujo tendrá como regente a cargo a Angel Guido quien, además de haber sido profesor de dibujo de los cursos vocacionales de la escuela en 1931 y 1932, era ya un reconocido arquitecto de la ciudad (9). Guido en su discurso inaugural esboza con claridad buena parte de los propósitos que adjudica a esta carrera y de sus ideas acerca de las artes en general. Señala que se trata del momento indicado para "consumar la Segunda Reconquista del Arte Americano frente al Europeo" (Guido, 1936: 15) y agrega que como primer jalón efectivo de esta fe americanista en el arte nuestro se crea este profesorado "inspirado, se diría, en la Eurindia de Ricardo Rojas, el limpio maestro de la argentinidad" y fruto de una "demanda estético-espiritual por un arte propio" (Guido, 1936, p. 16) (10). En referencia a la función específica de formar docentes apunta Guido que "no se trata de un Instituto de enseñanza más, sino de una escuela para maestros, es decir, una escuela de formación estética del futuro niño argentino" (Guido, 1936: 16. La negrita es nuestra). Insiste Guido en este discurso en resaltar además la localización geográfica e institucional de esta carrera:

Para la creación de este Profesorado de Arte Neocriollo -si se nos permite la expresión- se ha elegido Rosario (...) la ciudad granero, la ciudad más preocupada en las especulaciones materiales del trabajo y del comercio que en las especulaciones del espíritu. Sin embargo, también en este sentido han tenido gran acierto los creadores de este profesorado. Rosario, a pesar de sus detractores consuetudinarios, está llamada a cumplir un gran destino de cultura en el país (...) No es secreto el afirmar que difícilmente habrá en todo el país una educadora eminente más capacitada para dirigir este flamante instituto de formación estética que la Srta. Dolores Dabat. Efectivamente, en la persona de la Srta. Dabat convergen maravillosamente aquellas altas condiciones pues, junto a su vocación apasionada por la enseñanza une una cultura brillante y excepcional, que le permite ejercer con gran capacidad la difícil dirección intelectual y espiritual de este Profesorado de Arte Neocriollo. (Guido, 1936, p.p. 18-19)

Estos discursos fundantes evidencian sin dudas la clave estética presente -y posiblemente predominante- en el profesorado de dibujo: la búsqueda de una modalidad expresiva autóctona, de una identidad estética propiamente 
"argentina" inscripta en la corriente americanista que aglutinó a una parte de los artistas e intelectuales de la época tanto como generó numerosos detractores y profundos enfrentamientos. De hecho, no es esta vertiente estética la que representa a las vanguardias artísticas locales que se encontraban, en muchos casos, en las antípodas del hispano-americanismo invocado por Guido (11).

En cuanto a los profesores de la carrera, entre los que se encontraban el mismo Angel Guido y la ya mencionada Lelia Echezarreta, es importante mencionar que fueron muchos de ellos artistas e intelectuales notables. Nombramos algunos: Demetrio Antoniadis, pintor paisajista de origen griego que participó en diversos salones de Rosario y Buenos Aires; Nicolás Russo o Nicolás de San Luis, tal como se lo conocía, pintor y escultor nacido en Italia, radicado más tarde en San Luis y luego en Rosario, formado en la Academia Nacional de Bellas Artes y en Europa; Juan de los Ángeles Naranjo, nació en Rosario, fue alumno de Pedro Blanqué, se perfeccionó en España, Italia y Estados Unidos, trabajó para la Metropolitan Opera House y, entre otras cosas, al volver a radicarse en el país, además de su tarea docente, se desempeñó como Director del Museo Municipal de Bellas Artes "Juan B. Castagnino"; Manuel Suero, pintor y grabador español, estudió en la Universidad de La Plata. Más tarde se suman como profesores otros intelectuales y artistas reconocidos como el crítico de arte Ricardo Montes i Bradley y el pintor Carlos Uriarte.

Además, en sus primeros años, el profesorado contó entre sus alumnos con quiénes luego serían reconocidos artistas locales, tales como Hugo Ottman, Osvaldo Traficante, María Asunción Alonso, Martha Bugnone, Clelia Barroso, entre otros. Para estímulo de los egresados del profesorado de dibujo se instituyó el Salón Primavera con diversos premios, Salón que se realizó en 1947 y 1948.

\section{El traspaso del Profesorado de Dibujo a la universidad}

Al promediar la década del 40 se estaba ya gestando el pasaje de estas carreras al ámbito de la universidad. En el año 1945, Angel Guido, todavía a cargo del profesorado de dibujo, prepara un proyecto para la creación de la Escuela Superior de Bellas Artes de Rosario anexa a la Universidad Nacional del Litoral, a pedido del entonces Ministro de Justicia e Instrucción Pública de la Nación Dr. Antonio J. Benítez. En ese proyecto, Guido establece como objetivos de esta futura escuela "la formación de artistas y artesanos" con el propósito de:

(...) crear una nueva fuente de riqueza en nuestra economía al sentar las bases de una industria artística típicamente argentina (...) mejorar y enaltecer el nivel estético del pueblo argentino (...) formar artistas de arte puro, espiritualmente hermanados con todos los pueblos de América (...) iniciar un gran movimiento cultural hacia el 
renacimiento de los oficios y gremios de obreros artistas argentinos. (Artículo publicado en el diario Crónica, 29 de septiembre de 1973).

En esta propuesta de Guido se destaca la intención de articular en la formación el arte y la artesanía, además critica la escasa jerarquía de las llamadas artes menores y la desestimación estética que supone relegar su enseñanza a las escuelas de artes y oficios reservando las academias para las bellas artes o para el arte puro. Señala Guido que esto trajo como consecuencia "el mal gusto y la falta de belleza en los objetos industrializados y manufacturados que el hombre del pueblo usa, vive y convive cotidianamente" y recuerda la "misión ético-social de la Belleza y su influencia en la salud espiritual de los pueblos" (Guido, 1947, p. 9).

El proyecto, complejo y ambicioso, postula una estructura de formación de tres niveles de tres años cada uno que comprendían: una Escuela Preparatoria a la que se ingresaría con sexto grado aprobado y con un examen de ingreso "vocacional" cuya titulación sería la de Bachiller en Bellas Artes; una Escuela Intermedia que expediría títulos de Profesor Normal Nacional de Dibujo -para egresados de Escuelas Normales- y de Profesor Nacional de Dibujo -para egresados de otras carreras, incluyendo la preparatoria-; una Escuela Superior que otorgaría el título de Licenciado Nacional en Bellas Artes. La estructura de formación se montaba sobre dos escuelas pre-existentes: la primera de ellas se localizaba en la Escuela de Artes Plásticas de la provincia de reciente creación en 1942, la segunda se ubicaba sobre el profesorado de la Escuela Normal $\mathrm{N}^{\circ} 2$ y la tercera se crearía en el marco de la Universidad Nacional del Litoral. Además proponía la creación de una Escuela Anexa de Artesanos que también dependería de la Universidad del Litoral y que otorgaría el título de Maestro Mayor en Bellas Artes. De este modo, el proyecto de Guido articula casi todas las experiencias sistemáticas de formación artística existentes en la ciudad hasta esa fecha secuenciándolas en pos de una formación que contemplara tanto la docencia, lo académico y lo artístico como lo artesanal y lo social.

Dos años más tarde, en 1947, durante la primera presidencia de J. D. Perón, el Congreso de la Nación crea por ley de presupuesto de ese año la Escuela Superior de Bellas Artes con sede en la ciudad de Rosario dependiente de la entonces Secretaría de Educación de la Nación. Ese mismo año el Dr. Belisario Gache Pirán, Ministro de Justicia e Instrucción Pública, nombra una Comisión honoraria de expertos para dar comienzo formal a esa escuela. La Comisión estaba integrada por Alfredo Guido, como delegado de ese Ministerio, Donato Proieto, Eduardo Darnes y el mismo Angel Guido. Esta Comisión eleva al Ministro un dictamen a mediados de ese mismo año basado sustancialmente en la citada publicación de Angel Guido. El documento señala que esta nueva Escuela Superior de Bellas Artes deberá ser similar a la Escuela Superior de Bellas Artes de la Nación "Ernesto de la Cárcova" -que estuvo dirigida entre 1932 y 1955 por Alfredo Guido- y que deberá tener por tanto su misma cate- 
goría y sus mismos programas de enseñanza técnica y artística (Expediente № 4547, Facultad de Filosofía, Letras y Ciencias de la Educación, Universidad Nacional del Litoral) (12).

Poco después, Angel Guido asume como Rector de la Universidad Nacional del Litoral y solicita la incorporación de la Escuela Superior recientemente creada a esa casa de estudios "conjuntamente con todo el patrimonio del material didáctico"; incorporación que se concreta a principios de 1949 y en cuyo decreto de traspaso se estipula la "orientación social y argentina" de la Escuela. En 1950 comienzan a dictarse los cursos universitarios con 45 alumnos inscriptos.

El tránsito de este profesorado en la universidad continúa cargado de conflictos: un nuevo interventor en la Universidad Nacional del Litoral designa como nuevo director de la Escuela a Juan Zecchi quien introduce modificaciones en el plan de estudios y suprime materias prácticas reemplazándolas por otras teóricas. Luego, con un nuevo cambio de autoridades universitarias, el Delegado Interventor de la Facultad de Filosofía y Letras de la UNL convoca a una Comisión Especial destinada a proyectar un plan de enseñanza para la Escuela Superior de Bellas Artes que dependía de esa unidad académica. Vale mencionar que integraban, junto a otros, esa Comisión el mismo Angel Guido y otros antiguos docentes del profesorado de dibujo de la escuela normal como Carlos Uriarte, Nicolás Russo y Manuel Suero. En el informe elaborado por esta Comisión se argumenta la propuesta en un estudio de todos los antecedentes y en la experiencia vivida precisamente por los integrantes de la misma como docentes del profesorado desde su creación en 1936 y en la Escuela Superior desde 1950. Finalmente la Comisión propone: un plan de estudios de cuatro años de duración para el Profesorado Nacional de Dibujo, un plan de estudios para la Escuela Superior de Bellas Artes de tres años de duración similar al de la Escuela Superior "Ernesto de la Cárcova" que otorgaría las titulaciones de "Profesor Nacional en Pintura, Escultura, Grabado y Decoración Mural y un plan de estudios para la Escuela de Artesanos que comenzaría a funcionar más adelante cuando se obtenga la correspondiente partida presupuestaria.

Entre tanto, en la Escuela Normal el traspaso del profesorado a la universidad fue vivido como una pérdida:

(...) transcurrieron como en un soplo los años de vida de este ideal pedagógico de Lola Dabat (...) nos lo dejó a nuestro cuidado, pero no pudimos retenerlo entre nuestras manos, en nuestra escuela (...) tal vez nos cegó la ambición de verlo crecer y crecer, demasiado y demasiado rápido, cuando aún era muy pronto para juzgar su valor de entonces y el futuro (...) lo dejamos ir (...) en vano fue que luego pidiéramos su retorno" (Shakespear, 1960, p. 82).

La percepción institucional es que este traspaso desvirtuó la finalidad para la que estas carreras habían sido creadas, esto es, la formación de profesores 
en ambas especialidades. Se intenta además infructuosamente durante los años que siguen recuperar estas carreras: "se realizan insistentes gestiones por la vía oficial tendientes a concretar su reimplantación en esta Casa" (Shakespear, 1960: 47). Reimplantación que no se produce puesto que, hasta el presente, las carreras permanecen en el ámbito de la actual Universidad Nacional de Rosario creada en 1968 sobre la base de la sede en Rosario de la Universidad Nacional del Litoral.

\section{Comentarios finales}

Decíamos al inicio que las primeras décadas del siglo XX constituyeron un tiempo nuevo, inaugural, en el que se alienta una transformación que se intuye inminente (Funes, 2006). En este aspecto, creemos que la notable actividad educativa y cultural que tuvo lugar entonces en esta escuela normal estuvo en consonancia con los debates pedagógicos y los climas estéticos propios de la época. Así, la escuela se caracterizó por una visión ampliada de la tarea educativa como "tarea de cultura" en cuyo marco se inscriben tanto la profusa cantidad de iniciativas culturales que en ella tuvieron su origen como las innovaciones pedagógicas escolanovistas que allí se realizaron.

De este modo, la apertura del profesorado de dibujo constituyó posiblemente la concreción en un proyecto educativo de las inquietudes estéticas presentes en la institución -o, al menos, en sus autoridades- que se articuló, por una parte, con la necesidad local derivada de la carencia de ámbitos públicos especializados en brindar formación artística en la ciudad y, por otra, con el proceso de institucionalización de la educación artística que en esas décadas se llevaba a cabo en el país. Fue esta la primera propuesta oficial de capacitación de profesores en artes de la ciudad y coincidieron en ella las expectativas -no siempre conciliables- de formar docentes y la de formar artistas.

Esta tensión se pone en evidencia en los matices diferentes que se registran y expresan en Dabat y Guido. En el discurso inaugural de la carrera Dolores Dabat reivindica al normalismo y a los docentes como agentes portadores y multiplicadores de cultura. Esto es, la implantación del profesorado en el ámbito de la escuela normal supuso, para Dabat, una opción estética y política destinada a fortalecer al magisterio y a democratizar el arte tornándolo patrimonio escolar.

Angel Guido, por su parte, alude a un proyecto estético para el país de corte americanista que implica una gesta -casi una cruzada- de "reconquista" del arte americano frente al europeo, fuente de la constitución de un arte "neocriollo" y "propiamente argentino". Así, el profesorado de dibujo asume para Guido una finalidad ambiciosa, está llamado constituirse en una "escuela de formación estética para el futuro niño argentino". No obstante, si bien reivindica inicialmente la decisión de instalar las carreras de profesorado en una escuela normal que se destaca por sus actividades culturales, las palabras de Guido 
revelan que su principal interés es precisamente sentar las bases para un arte americano y nacional y que para ello requiere de instituciones más especializadas. De hecho, en pos de jerarquizar los estudios, impulsa y concreta el pasaje del profesorado al ámbito universitario.

Sin dudas se esbozan aquí tensiones cuyo análisis sería pertinente profundizar: entre la formación de docentes y la de artistas; entre las instituciones oficiales especializadas en una y otra; entre posiciones divergentes dentro del campo mismo del arte local. De la misma manera, se vislumbran en esta iniciativa y su devenir cuestiones propias del proceso de especialización creciente de la formación docente en el país, que va generando trayectos disciplinares diferenciados frente a la formación generalista que caracterizaba al magisterio normal.

Más allá de estos matices, es posible sostener que en la creación de esta carrera se concreta una articulación que resulta altamente fecunda en cuanto da lugar a un intenso movimiento que conjuga pedagogía, arte y cultura y que convoca tanto a pedagogos como a intelectuales y artistas a un diálogo cuya marca inaugural permanece hasta el presente en instituciones y experiencias que recuperan, subvierten y refundan esta herencia.

\section{Notas Bibliográficas}

(1) Con institucionalización nos referimos al proceso a través del cual se confiere a la educación artística carácter formal al interior de la estructura del Estado. Este proceso supone al menos dos cuestiones: la sistematización y selección de los saberes del campo artístico que serán oficializados mediante su inclusión en la educación oficial y la certificación que acredita el pasaje por estos trayectos educativos.

(2) Recién en 1942 comenzará a funcionar la Escuela Provincial de Bellas Artes (actual Escuela Provincial de Artes Visuales). En 1945 se creará también la Escuela Municipal Manuel Musto en lo que había sido la casa del pintor, a instancias de lo dispuesto por él en su testamento.

(3) Algunas investigaciones que se refieren a las experiencias escolanovistas desarrolladas en esta institución pueden consultarse en: Carli, 2002; Miguel - Laurino - Gonzalez, 2001; Ziperovich, 1992 en Puiggrós, 1992. Aunque no constituye el objeto del presente trabajo, es interesante mencionar que encontramos en esta institución dos etapas distintas en lo que hace a la implementación de ideas pedagógicas renovadoras. La primera, más utópica, auspiciada por Martín Herrera y vinculada a los precursores del movimiento escolanovista como Pestalozzi, Tolstoi o Rousseau, que se observa en la Escuela al Aire Libre, de escasa sistematicidad y de fuerte contenido social. Probablemente -y así lo afirma por ejemplo Ziperovich en las páginas que dedica a esta experiencia- hay aquí una estrecha vinculación también con las ideas pedagógicas anarquistas, particularmente en la figura de Haydée Maciel. En este sentido, podríamos decir que se trata de una iniciativa que en sus comienzos asume un carácter rupturista y fuertemente radicalizado (Puiggrós, 1992) que finalmente se diluye y se integra al conjunto del sistema educativo (Maciel, 1951). La segunda etapa, impulsada por Dolores Dabat desde la dirección de la escuela, más elaborada desde el punto de vista didáctico, inscripta en las ideas es- 
colanovistas de las primeras décadas del siglo, y particularmente de modo explícito en las ideas y propuestas de Decroly, pero articulada orgánicamente al modelo normalista y al funcionamiento escolar estandarizado (Puiggrós, 1992).

(4) Algunos autores coinciden en señalar que el ideario del escolanovismo contribuyó a la maduración teórica acerca de la educación estética así como a la expansión de experiencias de ese tipo (Gennari, 1997; Wojnar, 1967).

(5) La Universidad inicia las clases el 21 de septiembre de 1918 con una inscripción que superó los 400 alumnos, se dictaban entonces cursos prácticos de corta duración en diversas ramas del comercio, las industrias y las artes (Mikielievich, 1972). Más tarde se anexa formalmente a la Universidad Nacional del Litoral, pasando a integrar el Instituto Social que se crea en esta en 1928 (Baraldi, 2007).

(6) El primer número de esta revista -cuyo análisis merecería un tratamiento aparte- se publica en abril de 1932 y que continúa editándose hasta 1936, fecha en la que debido a problemas económicos deja de circular. Entre sus tópicos y desde la tapa misma la publicación se anuncia como revista de pedagogía, literatura, ciencia y arte. Si bien se destaca la colaboración en la revista de personajes reconocidos con diversos tipos de textos como Celia Ortiz de Montoya, Bernardina Dabat, Hugo Calzetti, Víctor Mercante, Mateo Booz, Guido Buffo, César Tiempo, Alvaro Yunque, José Pedroni, Antonio Sagarna, Juan Alvarez o Ricardo Rojas entre otros, lo que resulta notable tratándose de una revista editada desde una institución escolar es la cuidada edición gráfica.

(7) Alfredo Guido fue reconocido como muralista en la década del 20 en la ciudad puesto que además de este mural enviado a la Exposición de Sevilla de 1929 realiza otros: uno para el Palacio de la Federación Agraria y otro para una residencia particular. Guido fue también director de la Escuela Superior de Bellas Artes de la Nación "Ernesto de la Cárcova" entre 1932 y 1955.

(8) Echezarreta fue además ilustradora de publicaciones especializadas del naturalista francés Jean Henry Fabre, una de ellas presentada en 1927, junto a otras dos obras, en el IV Salón del Museo Rosa Galisteo de Rodríguez de la ciudad de Santa Fe.

(9) La obra más popular de Angel Guido fue y es sin dudas el Monumento a la Bandera, localizado en la ciudad de Rosario, frente al río Paraná. Guido nace en Rosario en 1898, estudia en la Universidad de Córdoba de donde egresa en 1920 como Ingeniero y en 1921 como Arquitecto. Fue docente de la Universidad Nacional del Litoral a poco de creada esta. En 1948 fue nombrado Rector de esta Universidad, cargo que desempeña durante dos años. Fue amigo de Ricardo Rojas y tomó de él concepciones referidas a la necesidad de superar el "aluvión extranjerizante" proponiendo valores culturales y estéticos propios. Muere en Rosario en 1960. Por otra parte, Guido publicó una importante cantidad de libros. De hecho al momento de asumir la dirección de esta carrera había publicado ya en 1925 "Fusión hispanoindígena en la Arquitectura Colonial", en 1927 "La Arquitectura hispanoamericana a través de Wolfflin" y "Orientación espiritual de la Arquitectura en América", en 1930 "La Machinolatrie de Le Corbusier" y "Eurindia en el arte hispanoamericano", en 1932 "Arqueología y estética de la Arquitectura criolla", "El arte de nuestro tiempo" y "Definición de la Reforma Universitaria", en 1936 "Catedrales y rascacielos" y "Concepto moderno de la historia del arte", entre otros.

(10) Angel Guido sostiene esta posición en otras instituciones de la ciudad. Desde 1939, año en que se inaugura el Museo Histórico Provincial, participa del mismo como secretario y asume la dirección artística de la Exposición de Arte Religioso Retrospectivo que se realiza en 1941. Vale apuntar que este museo desde su creación se abocó a la "búsqueda de nuestra nacionalidad en la fusión hispano-indígena" (Montini, 2008 en Artundo - Frid, 2008) 
(11) Recordemos que en la década del 30 se produce en la ciudad la irrupción de un movimiento artístico de vanguardia. En 1933 se conforma la Mutualidad de Artistas Plásticos, encabezada por Antonio Berni recién llegado de París, que asumió una posición política radicalizada y una estética vanguardista distante de los planteos americanistas de Guido.

(12) La Escuela Superior de Bellas Artes de la Nación había sido creada en 1921, durante el gobierno de Hipólito Irigoyen a instancias de Ernesto de la Cárcova quien fuera además su primer director y cuyo nombre asume en 1928 después de la muerte del artista en 1927. Alfredo Guido asume la dirección de esta escuela en 1932 y permanece allí durante más de veinte años no exentos de conflictos políticos e ideológicos.

\section{Referencias bibliográficas}

- $\quad$ Alvarez, J. (1998). Historia de Rosario (1689 - 1939). Rosario: UNR Editora.

- $\quad$ Artundo, P. (2008). Arte en revistas. Publicaciones culturales en la Argentina $1900-$ 1950. Rosario: Beatriz Viterbo.

- $\quad$ Artundo, P. (2006). Modernidad y vanguardia en la década del veinte. Ponencia presentada en el Simposio sobre el arte en la modernidad y en la contemporaneidad. San Pablo, Brasil.

- $\quad$ Artundo, P. - Frid, C. (2008). El coleccionismo de arte en Rosario. Colecciones, mercado, exhibiciones, 1880 - 1970. Buenos Aires: Fundación Espigas.

- Baraldi, V. (2007). Articulación educación - sociedad en los albores de la creación de la Universidad Nacional del Litoral. Ponencia presentada en el V Encuentro Nacional y II Latinoamericano "La Universidad como objeto de investigación", Universidad Nacional del Centro de la Provincia de Buenos Aires, Tandil.

- $\quad$ Bürger, P. (1987). Teoría de la Vanguardia. Barcelona: Península.

- $\quad$ Carli, S. (2002). Niñez, pedagogía y política. Buenos Aires: Miño y Dávila.

- Carli, S. (1992). El campo de la niñez. Entre el discurso de la minoridad y el discurso de la Escuela Nueva. En Puiggrós, A. (dir.) Escuela, Democracia y Orden (1916 - 1943). Buenos Aires: Galerna.

- Córdoba Lutges, María A. B. de (1972). Las artes plásticas en Rosario. Historia de las Instituciones de la Provincia de Santa Fe, Tomo V, $1^{\circ}$ parte. Santa Fe: Edición oficial.

- Fantoni, G. (1993). Itinerario de una modernidad estética. Intensidades vanguardistas y estrategias de modernización en el arte de Rosario. Arte y Poder. Buenos Aires: CAIA, Buenos Aires.

- Fernández, S. (2010). La revista El Círculo o el arte de papel. Murcia: Universidad de Murcia, Servicio de Publicaciones.

- Funes, P. (2006). Salvar la nación. Intelectuales, cultura y política en los años veinte latinoamericanos. Buenos Aires: Prometeo.

- Gennari, M. (1997). La educación estética. Barcelona: Paidós.

- Giunta, A. (2004). La sociedad de los artistas de Rosario. Rosario: Museo Municipal de Bellas Artes Juan B. Castagnino.

- Gschwind, J. (1950). Tres capítulos para la historia de la educación de Rosario. Rosario: Academia Nacional de Historia.

- $\quad$ lelpi, R. (2005). Rosario, del 900 a la década infame, Tomos I, II, III y IV. Rosario: Homo Sapiens.

- Miguel, A. de - Laurino, E. - Gonzalez, I. (2001). Dolores Dabat y una escuela para "ser feliz". Ponencia presentada en las XII Jornadas de Historia de la Educación, Rosario. 
- Mikielievich, W. (1972). La instrucción pública en Rosario. Historia de las Instituciones de la Provincia de Santa Fe, Tomo V, $1^{\circ}$ parte. Santa Fe: Edición oficial.

- Mouguelar, L. (2002). Los murales de Julio Vanzo. Un acercamiento al modernismo en Rosario. Revista Separata, Centro de Investigaciones del Arte Argentino y Latinoamericano, Facultad de Humanidades y Artes, Universidad Nacional de Rosario, Año II, Números 3 y 4.

- $\quad$ Pacheco, M. Telesca, A. M. (1988). La pintura y el ambiente artístico argentino en la década del veinte. Antología documental. Buenos Aires: Universidad de Buenos Aires, Facultad de Filosofía y Letras.

- Pineau, P. (2007). Cuadros de una exposición: comentarios sobre la escuela como máquina estetizante. En Frigerio, G. - Diker, G. Educar: (sobre) impresiones estéticas. Buenos Aires: Del Estante Editorial.

- Peña, F (2008). Amigos del cine. Amigos del Arte 1924 - 1942. Buenos Aires: Malba Fundación Constantini.

- $\quad$ Plá, A. (coord.) (2000). Rosario en la Historia, Tomo I. Rosario: UNR Editora.

- $\quad$ Puiggrós, A. (dir.) (1992). Escuela, Democracia y Orden (1916 - 1943). Buenos Aires: Galerna.

- $\quad$ Puiggrós, A. (dir.) (2001). La Educación en las Provincias y Territorios Nacionales (1885 - 1945). Buenos Aires: Galerna.

- Sarlo, B. (1988). Una modernidad periférica: Buenos Aires 1920 y 1930. Buenos Aires: Nueva Visión.

- Sendra, R. (1990). Rosario, ciudad y artes plásticas. Rosario: Dirección de Publicaciones UNR.

- $\quad$ Slullitel, I. (1968). Cronología del arte en Rosario. Rosario: Edición del autor.

- Wojnar, I. (1967). Estética y pedagogía. México: Fondo de Cultura Económica.

\section{Fuentes y documentos consultados}

- Dabat, D. (1935). Homenaje a la Escuela Normal N² 1910 - 1935. Rosario: Publicaciones de Quid Novi.

- Dabat, D. (1936). Discurso Inauguración de los cursos de Profesorado de Música y Dibujo. Quid Novi? Suplemento, Tomo V, № 28.

- Guido, A. (1936). Discurso Inauguración de los cursos de Profesorado de Música y Dibujo. Quid Novi? Suplemento, Tomo V, № 28.

- Guido, A. (1947). Academia Superior de Bellas Artes de Orientación Social para Rosario. Rosario: Edición del autor.

- $\quad$ Shakespear, I. (dir) (1960). Homenaje a la Escuela Normal N² 1910 - 1960. Rosario.

- 90 años, Reseña histórica Escuela Normal Superior N² Provincial N 35 "Juan María Gutierrez", Rosario, 2000.

- Maciel, H. (1951). La escuela al aire libre, ensayo tolstiano, en Martín Herrera. Su vida, su obra. Rosario.

- Memoria del Instituto Social 1928- 1932 (escrita por Juan Alvarez). Universidad Nacional del Litoral, Santa Fe, 1934.

- Memoria Instituto Social 1933 - 1936. Universidad Nacional del Litoral, Santa Fe, 1937.

- Memoria Instituto Social 1937 - 1940. Universidad Nacional del Litoral, Santa Fe, 1937. 
- Documentos de la Asociación de Ex-Alumnas de la Escuela Normal № 2 "Ana María Benito".

- $\quad$ Revistas Quid Novi? Año I, Números 1, 2, 3 y 4 (1932) y Año II, Números 5 y 6 (1933).

- Expediente $\mathrm{N}^{\circ}$ 4547, Facultad de Filosofía, Letras y Ciencias de la Educación, Universidad Nacional del Litoral. 